\title{
Simultaneous Position-Resolved Mapping of Chromatic Dispersion and Brillouin Shift Along Single-Mode Optical Fibers
}

\author{
Miguel González-Herráez and Luc Thévenaz
}

\begin{abstract}
We describe a new method for performing simultaneous position-resolved measurements of chromatic dispersion and Brillouin shift along single-mode optical fibers. The technique provides consistent high-resolution and low-noise results for both quantities. Our measurements indicate a certain correlation between both magnitudes, although the degree of correlation varies for the different manufacturers tested.
\end{abstract}

Index Terms-Brillouin, chromatic dispersion, distributed measurements.

\section{INTRODUCTION}

$\mathbf{N}$ ONLINEAR transmission in optical fibers is governed not only by the average (end-to-end) value of chromatic dispersion along the fiber, but also by the local fluctuations of the dispersion coefficient. In one side, random variations of this coefficient cause irreversible broadening of the soliton [1] and, thus, an increase in the bit-error rate. On the other side, recent experiments have shown that if the dispersion distribution is adequately designed and controlled (dispersion management), strong improvement can be found with respect to the traditional approaches in pulse transmission, specially regarding four-wave mixing (FWM) issues [2]. As a result of this potential benefit, several methods for mapping chromatic dispersion fluctuations in optical fibers have already been presented in the literature [3]-[6]. This letter presents a new method for simultaneous position-resolved measurement of chromatic dispersion and Brillouin shift along an optical fiber. This new technique does not only overcome some limitations of previous chromatic dispersion mapping techniques but also might reveal the cause of the chromatic dispersion variations along the fiber by relating them to strain or doping concentration variations. Moreover, our preliminary measurements suggest a correlation between both magnitudes. This correlation is found to depend on the manufacturing process used.

\section{MEASUREMENT PRINCIPLE}

We consider two pumps $\left(f_{I}<f_{K}\right)$ delivering a few milliwatts $(5-10 \mathrm{~mW})$ of power at the fiber input. By means of an FWM process, a Stokes and an anti-Stokes wave will

Manuscript received October 24, 2003; revised November 21, 2003. This work was supported by the Swiss Federal Office for Education and Science.

The authors are with the Nanophotonics and Metrology Laboratory, École Polytechnique Féderal de Lausanne, Lausanne CH-1015, Switzerland (e-mail: miguel.gonzalez@epfl.ch).

Digital Object Identifier 10.1109/LPT.2004.824926 be generated along the fiber, following energy conservation rules $\left(f_{S}=2 f_{I}-f_{K}, f_{A}=2 f_{K}-f_{I}\right)$. The power of the anti-Stokes wave oscillates along the fiber with a spatial frequency $\Delta \beta=2 \beta\left(f_{K}\right)-\beta\left(f_{I}\right)-\beta\left(f_{A}\right)$, where $\beta$ stands for the propagation constant. If the frequency difference between the two pumps is small, the term $\Delta \beta$ can be directly related to the group velocity dispersion by the expression $\Delta \beta=\beta_{2}\left(f_{K}\right)\left(\omega_{K}-\omega_{I}\right)^{2}$, where $\beta_{2}$ is the second derivative of the propagation constant with respect to the frequency. Similar rules apply for the Stokes wave. The basic idea for the local measurement of chromatic dispersion is to retrieve the power distribution of the Stokes or anti-Stokes wave along the fiber. With this power distribution and a simple time-frequency analysis, it is possible to retrieve the dispersion distribution along the fiber. A plain optical time-domain reflectometer-like configuration for this measurement has been demonstrated. Since the FWM conversion efficiency is small, very high FWM pump powers $(\sim 1 \mathrm{~W})$ are required. A different approach using drastically smaller continuous-wave FWM pump powers was proposed in [6], that prevents any risk of modulation instability from arising in the presence of small and positive dispersion. Since the pumps are continuous-wave, in this setup, the powers must be kept below the Brillouin threshold $(<10 \mathrm{~mW})$. The method for retrieving the FWM power distribution is based on performing Brillouin optical time-domain analysis (BOTDA) over the FWM signal. Since stimulated Brillouin scattering can be made orders of magnitude more efficient than Rayleigh backscatter, smaller values of FWM pump powers are required. For the BOTDA configuration, a spectrally narrow pump pulse is launched into the fiber in opposite direction. The optical frequency of the Brillouin pump pulse has to be approximately $f_{A}+\nu_{B}$, where $\nu_{B}$ is the Brillouin shift. For this configuration, the signal obtained at the BOTDA fiber end (tuned at $f_{A}$ ) can be expressed as

$$
\Delta P_{A}(z)=\frac{g(\nu)}{A_{\mathrm{eff}}} P_{A}(z) P_{P}(z) \Delta z
$$

where $g(\nu)$ is the Brillouin linear gain (dependent on the frequency separation between FWM and Brillouin pump), $A_{\text {eff }}$ the mode effective area, $P_{A}$ and $P_{P}$ the powers of the anti-Stokes and Brillouin pump, respectively, and $\Delta z$ the pump pulse length. Since the Brillouin pump power is only subject to standard attenuation and, thus, varies very slowly, the amplified part of the signal $\Delta P_{A}(z)$ is essentially proportional to the local power $P_{A}(z)$ of the anti-Stokes signal. In conventional fibers, 


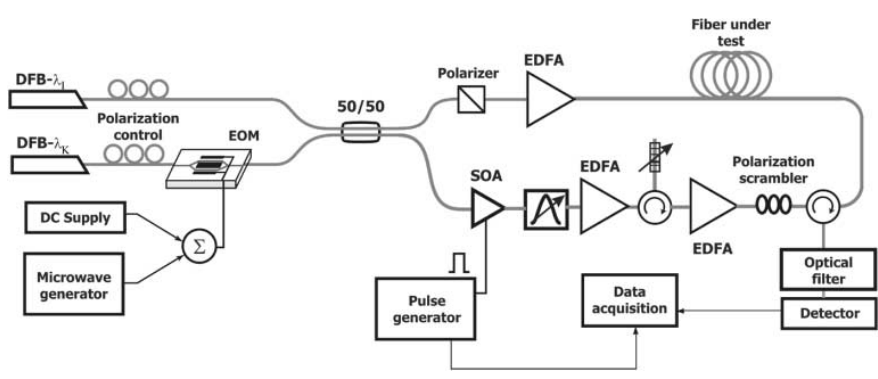

Fig. 1. Experimental setup. Typical pulse length is $1 \mu \mathrm{s}$.

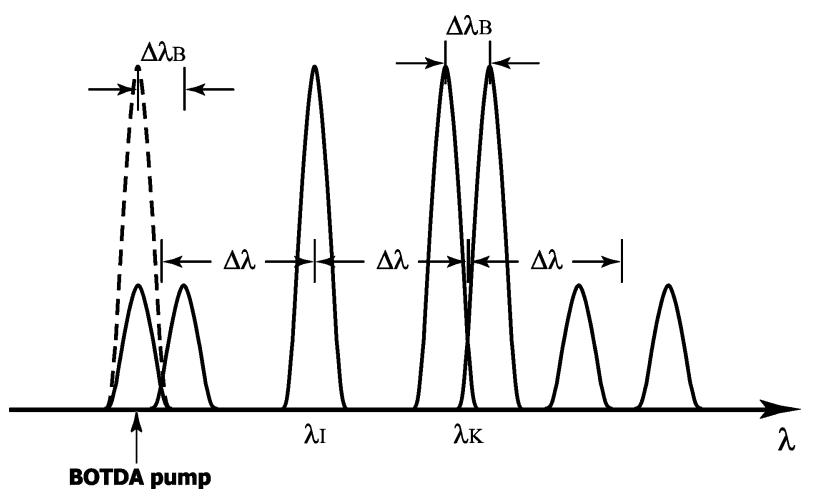

Fig. 2. Wavelength arrangement. $\Delta \lambda_{B}$ is the Brillouin shift.

$g(\nu)$ has a narrow lorentzian shape with a maximum at $\nu_{B}$ $(10.2-10.9 \mathrm{GHz}$ at $1550 \mathrm{~nm})$ and a full-width at half-maximum $\Delta \nu_{B}$ (about $25 \mathrm{MHz}$ at $1550 \mathrm{~nm}$ ). In [6], two lasers generated the FWM pumps and the BOTDA pump pulse was generated from a third different laser. The main drawback of this setup is that the evolution of the three frequencies is not completely fixed, and therefore, the analyzed FWM sideband might fall outside the Brillouin gain spectrum of the fiber. In this letter, we present a significant improvement of this method, in which the Brillouin pump is generated by modulation and FWM, resulting in an ideally stable frequency difference between Brillouin pump and anti-Stokes FWM signal $(\nu)$ for any wavelength separation between FWM pumps. Thus, by varying $\nu$, it is possible to simultaneously retrieve the power distribution of the anti-Stokes signal and to measure the Brillouin shift (the maximum Brillouin interaction is achieved when $\left.\nu=\nu_{B}\right) . \nu_{B}$ might vary along the fiber due to strain, temperature or doping concentration [7].

\section{EXPERIMENTAL SETUP AND RESULTS}

The experimental setup that we used is depicted in Fig. 1. In Fig. 2, we show the wavelength arrangement. Two tunable, distributed feedback lasers are used to generate the FWM pumps. The first pump is continuous-wave, while the second one is modulated, producing as a result two nearly symmetric sidebands separated approximately by the Brillouin shift. An electronic feedback control at the output of the electrooptical modulator (EOM) ensures that no carrier goes through, even in the case of thermal changes in the device [7]. One of the two sidebands is used (together with the first FWM pump) to generate the initial seed of the Brillouin pump pulse by FWM in a highly

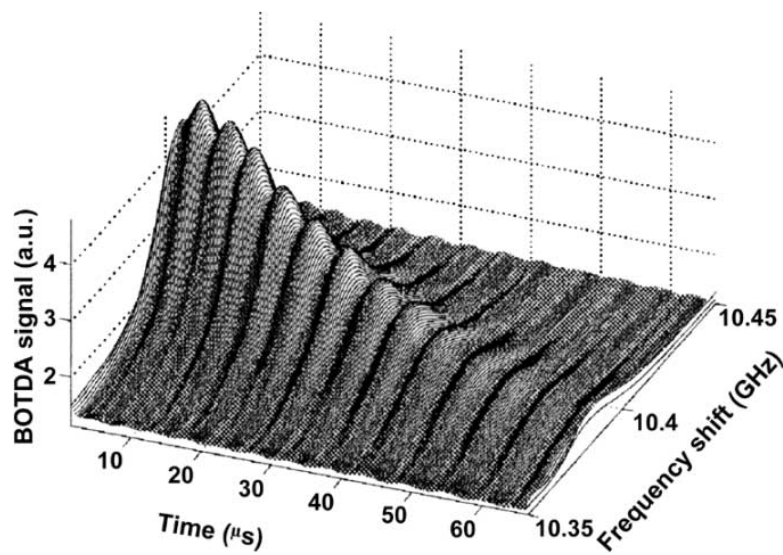

Fig. 3. Sample trace obtained with the system for a dispersion-shifted fiber with a measured end-to-end dispersion of $1.1 \mathrm{ps} \cdot \mathrm{nm}^{-1} \cdot \mathrm{km}^{-1}$. The wavelength separation between the two FWM pumps is $3.9 \mathrm{~nm}$, corresponding to a spatial period of approximately $480 \mathrm{~m}$.

nonlinear semiconductor optical amplifier (SOA). The pulse is shaped by gain switching of the SOA. The other sideband is used as a pump for the FWM process in the fiber. Thus, the frequency separation between the Brillouin pump and the FWM product generated in the fiber is constant (even in the case of random jitter in the frequency of the two distributed feedback lasers) and tunable (by acting on the RF generator). The two FWM pumps are aligned and amplified to saturation before entering the fiber.

The Brillouin pump pulse is subsequently filtered (with a tunable thin-film filter first, and a strain-tunable fiber Bragg grating later) and amplified with high-gain erbium-doped fiber amplifiers, to raise its power from a few microwatts to a few hundred milliwatts. The Brillouin pump pulse is introduced in the fiber in counterpropagating direction, thus, producing local Brillouin gain of the FWM product along the fiber. This gain can be observed at the input end of the Brillouin pump pulse by a suitable detector and a digitizing oscilloscope, like in any other BOTDA setup. The polarization of the Brillouin pump pulse is scrambled, and the acquired traces are averaged several thousand times to ensure that no polarization-dependent gain is recorded in the trace. The frequency separation between the Brillouin pump pulse and the FWM is swept all over the region of Brillouin gain of the fiber. The final result is a three-dimensional representation like the experimental results shown in Fig. 3. Once the Brillouin shift for each point is determined, we compute the power distribution of the anti-Stokes FWM signal. Over this power distribution along the fiber, we compute a spectrogram. The window of analysis gives the final resolution and uncertainty of the chromatic dispersion map. Bigger windows ensure less resolution but also a smaller uncertainty (bigger windows include more signal periods and, thus, a much more robust determination of the local oscillation). To figure out the precision of the local measurement, we have estimated that using a single period in the analysis yields an uncertainty in the spatial frequency determination of approximately $\pm 5 \%$.

Fig. 4 shows the final chromatic dispersion maps together with the corresponding measured Brillouin shifts for a dispersion-shifted fiber and a nonzero dispersion-shifted fiber. The experimental results from both sides are consistent, and in very 


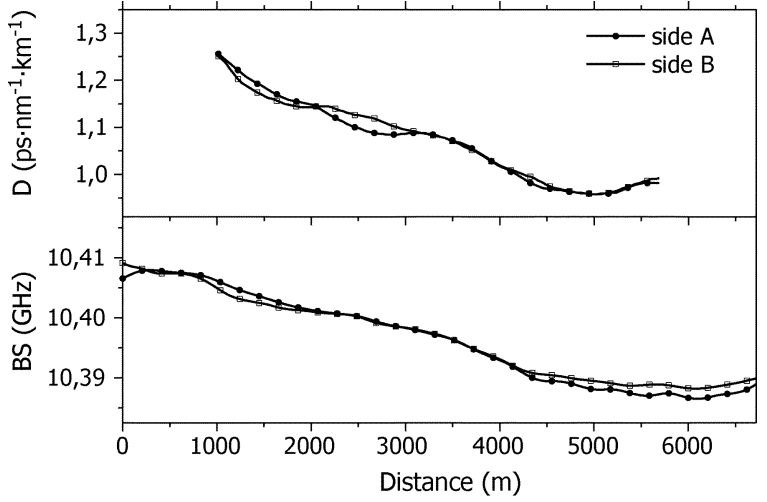

Fig. 4. Calculated chromatic dispersion map and Brillouin shift of the dispersion-shifted fiber from Manufacturer A quoted in Fig. 3. The analysis window is $2 \mathrm{~km}$ wide ( $\simeq 5$ signal periods) to provide a very low uncertainty in the local value of the dispersion.

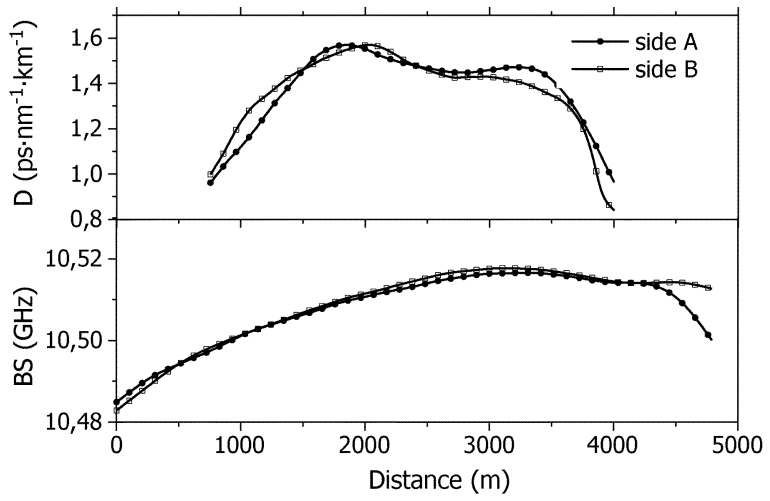

Fig. 5. Calculated chromatic dispersion map and Brillouin shift of a dispersion-shifted fiber from Manufacturer B with a measured end-to-end dispersion of $\sim 1.2 \mathrm{ps} \cdot \mathrm{nm}^{-1} \cdot \mathrm{km}^{-1}$ at the wavelength of interest. The wavelength separation between the two FWM pumps is $\sim 3.9 \mathrm{~nm}$. The analysis window is $1.5 \mathrm{~km}$ wide.

good agreement with end-to-end measurements. Visually, a certain correlation between the evolution of the dispersion coefficient $(D)$ and the Brillouin shift (BS) appears quite evident. Other measurements in nonzero dispersion-shifted fibers from the same manufacturer show a similar correlation between the two magnitudes, although there seems to be no direct translation from one magnitude to the other.

To verify this correlation, we performed the same measurements in dispersion-shifted fibers from other different manufacturers. Fig. 5 shows the chromatic dispersion and Brillouin shift distribution along a fiber from Manufacturer B. The correlation is less evident in this case, as in all the fibers measured from this manufacturer. The tested fiber spools from Manufacturers $\mathrm{C}$ and $\mathrm{D}$ showed no significant chromatic dispersion variation, as well as no significant change in the Brillouin shift along the fiber. All the standard dispersion fibers tested (from all the manufacturers) showed no significant variation of the dispersion as well as rather negligible variations in the Brillouin shift.

Regarding the dynamic range of the instrument, we have achieved successful mapping of a $\sim 20$-km-long concatenations of SMF fibers. In Fig. 6, we show the acquired trace for a concatenation of an 11.8-km-long fiber with $19 \mathrm{ps} \cdot \mathrm{nm}^{-1} \cdot \mathrm{km}^{-1}$ and a $4.5-\mathrm{km}$-long fiber with $16.2 \mathrm{ps} \cdot \mathrm{nm}^{-1} \cdot \mathrm{km}^{-1}$. The retrieved values of chromatic dispersion coefficient and Brillouin

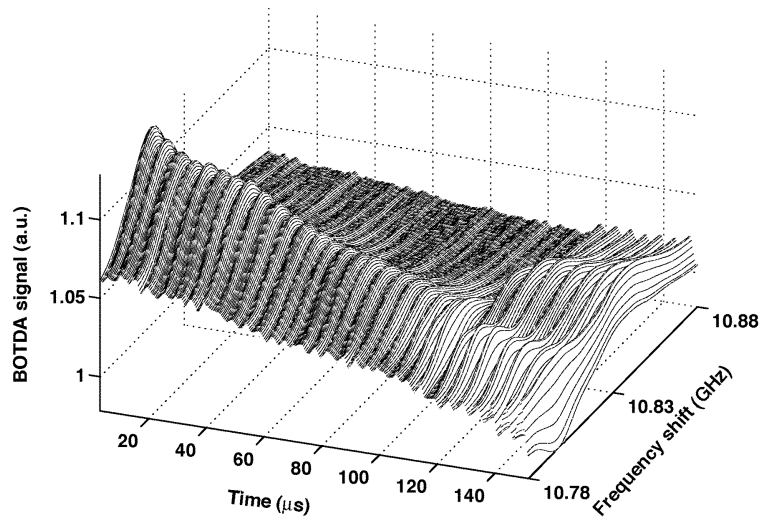

Fig. 6. Measured trace for a concatenation of the two standard fibers quoted in the text. The wavelength separation between the two FWM pumps is $\simeq 1 \mathrm{~nm}$. The spatial period recorded is $\simeq 420 \mathrm{~m}$ for the $19-\mathrm{ps} \cdot \mathrm{nm}^{-1} \cdot \mathrm{km}^{-1}$ fiber and $\simeq 500 \mathrm{~m}$ for the $16.2-\mathrm{ps} \cdot \mathrm{nm}^{-1} \cdot \mathrm{km}^{-1}$ fiber.

shift are in excellent agreement with end-to-end measurements. We believe that a suitable improvement of some still unoptimized aspects of the system (namely increasing the trace averaging and reducing the detection bandwidth) would lead to a superior performance of the dynamic range. With these improvements, we expect to achieve a dynamic range similar to that of a conventional BOTDA setup, around $50 \mathrm{~km}$.

\section{CONCLUSION}

An experimental technique for the simultaneous measurement of local chromatic dispersion and Brillouin shift along an optical fiber has been demonstrated. The experimental results are consistent and in good agreement with end-to-end data. Preliminary results show a correlation between both quantities in certain kinds of fibers. Further work and modeling should provide more insight into this correlation.

\section{ACKNOWLEDGMENT}

The authors would like to thank P. Corredera from the Instituto de Fisica Aplicada in Madrid (Spain) for providing some measured fibers.

\section{REFERENCES}

[1] M. Chertkov, I. Gabitov, P. M. Lushnikov, J. Moeser, and Z. Toroczkai, "Pinning method of pulse confinement in optical fiber with random dispersion," J. Opt. Soc. Amer. B, vol. 19, no. 11, pp. 2538-2550, 2002.

[2] L. F. Mollenauer, P. V. Mamyshev, J. Gripp, M. J. Neubelt, N. Mamysheva, L. Gruner-Nielsen, and T. Veng, "Demonstration of massive wavelength-division multiplexing over transoceanic distances by use of dispersion-managed solitons," Opt. Lett., vol. 25, pp. 704-706, 2000.

[3] L. F. Mollenauer, P. V. Mamyshev, and M. J. Neubelt, "Method for facile and accurate measurement of optical fiber dispersion maps," Opt. Lett., vol. 21, pp. 1724-1726, 1996.

[4] M. Eiselt, R. M. Jopson, and R. H. Stolen, "Nondestructive positionresolved measurement of the zero-dispersion wavelength in an optical fiber,” J. Lightwave Technol., vol. 15, pp. 135-143, Jan. 1997.

[5] I. Brener, P. P. Mitra, D. D. Lee, D. J. Thomson, and D. L. Philen, "Highresolution zero-dispersion wavelength mapping in single-mode fiber," Opt. Lett., vol. 23, pp. 1520-1522, Oct. 1998.

[6] M. Gonzalez-Herraez, L. Thevenaz, and P. Robert, "Distributed measurement of chromatic dispersion using four-wave mixing and Brillouin opticaltime domain analysis," Opt. Lett., vol. 28, pp. 2210-2212, 2003.

[7] M. Niklés, L. Thévenaz, and P.Ph. Robert, "Brillouin gain spectrum characterization in single-mode optical fibers," J. Lightwave Technol., vol. 15, pp. 1842-1851, Oct. 1997. 